\title{
S1 Text: Stability of working memory in continuous attractor networks under the control of short-term plasticity
}

\author{
Alexander Seeholzer ${ }^{1}$, Moritz Deger $^{1,2}$, Wulfram Gerstner ${ }^{1^{*}}$ \\ 1 School of Computer and Communication Sciences and School of Life Sciences, Brain Mind Institute, \\ École Polytechnique Fédérale de Lausanne, Lausanne, Switzerland \\ 2 Institute for Zoology, Faculty of Mathematics and Natural Sciences, University of Cologne, Cologne, \\ Germany \\ * wulfram.gerstner@epfl.ch
}

\section{Left eigenvector projects onto center positions}

Here, we provide additional details to the derivation of the relation Eq (16). Let the full diagonalization ${ }^{1}$ of the matrix $K$ be $\bar{K}=T^{-1} K T$. Without loss of generality, let $e_{r}$ be the first column of the transformation matrix $T$, and let $e_{l}$ be the first row vector of $T^{-1}$, with $e_{l} \cdot e_{r}=1$. Now, the projection of the perturbation $\delta \mathbf{y}$ onto contributions along $e_{r}$ isolates displacements of the center position: the perturbation $\delta \mathbf{y}=e_{r} \delta \varphi$ corresponds to a shift $\varphi+\delta \varphi$ in the center position (by definition, see Eq (14)). We left multiply this relation with $e_{l}$ to see that $e_{l} \delta \mathbf{y}=e_{l} e_{r} \delta \varphi=\delta \varphi$. Finally, we take the time derivative, which yields:

$$
\dot{\delta \varphi}=e_{l} \dot{\delta} \mathbf{y}
$$

Thus, to find an equation for the temporal dynamics of changes in the center positions, we project the system Eq (13) onto contributions along the $e_{r}$ eigenspace by left multiplying by $e_{l}$. In the full matrix equations, this corresponds to rewriting the system Eq (13) as

$$
T^{-1} \dot{\delta} \mathbf{y}=\bar{K} T^{-1} \delta \mathbf{y} .
$$

Restricting this onto only the first dimension (the $e_{r}$ eigenspace) and using Eq (S1), we find that the linear dynamics vanish (since the first entry in $\bar{K}$ is zero) and we are left with:

$$
\dot{\varphi}=\dot{\delta} \varphi=e_{l} \dot{\delta} \mathbf{y}=0 \cdot e_{l} \delta \mathbf{y}
$$

where we have assumed that $\varphi(t)=\varphi(t=0)+\delta \varphi(t)$.

Thus, left-multiplying the linearized equations Eq $(13)$ by $e_{l}$ yields a differential equation for the changes $\delta \varphi$ of the center position.

\section{Derivation of the left eigenvector}

We will find a parametrized vector $\mathbf{y}^{\prime}(\mathbf{y})=\left(\mathbf{t}^{T}(\mathbf{y}), \mathbf{v}^{T}(\mathbf{y}), \mathbf{z}^{T}(\mathbf{y})\right)^{T}$ that for $\mathbf{y}=e_{r}$ fulfills the transposed eigenvalue equation of the left eigenvector:

$$
K^{T} \mathbf{y}^{\prime}\left(e_{r}\right)=0 .
$$

\footnotetext{
${ }^{1}$ Or Jordan normal form, if $K$ is not fully diagonalizable. Since we know there exists a zero eigenvalue with one dimensional Eigenspace, the corresponding normal form will have a diagonal 0 entry with $e_{r}$ being the corresponding eigenvector.
} 
The system of equations resulting from $\dot{\mathbf{y}}^{\prime}=K^{T} \mathbf{y}^{\prime}$ reads (cf. Eq 13)):

$$
\begin{aligned}
\dot{t}_{i} & =-\frac{t_{i}}{\tau_{s}}+\sum_{j} w_{j i} u_{0, j} x_{0, j} \phi_{j}^{\prime} t_{j}+U \sum_{j} w_{j i}\left(1-u_{0, j}\right) \phi_{j}^{\prime} v_{j}-\sum_{j} w_{j i} u_{0, j} x_{0, j} \phi_{j}^{\prime} z_{j} \\
\dot{v}_{i} & =-\frac{v_{i}}{\tau_{u}}+\phi_{0, i} x_{0, i}\left(t_{i}-z_{i}\right)-U \phi_{0, i} v_{i} \\
\dot{z}_{i} & =-\frac{z_{i}}{\tau_{x}}+u_{0, i} \phi_{0, i}\left(t_{i}-z_{i}\right)
\end{aligned}
$$

Let us first consider the system without facilitation or depression. The linearized dynamics of $\delta$ s are given by the upper left $N \times N$ block $K_{s}$ of Eq 113 ), with $U \rightarrow 1, u_{0} \rightarrow 1, x_{0} \rightarrow 1$ (which recovers the case of [1]):

$$
\begin{aligned}
\delta \dot{s}_{i} & =-\frac{1}{\tau_{s}} \delta s_{i}+\phi_{0, i}^{\prime} \sum_{j} w_{i j} \delta s_{j} \\
& \equiv K_{s} \delta \mathbf{s}
\end{aligned}
$$

We assume that $w_{j i}=w_{i j}$, which in general holds for the symmetric synaptic connectivity of common models of continuous attractor networks (see below a concrete spiking model). The transposed block $K_{s}^{T}$ then describes the linear dynamics of perturbations to the input variables $J_{i}=\sum_{j} w_{i j} s_{j}[1$. To see this, we differentiate $\delta J_{i}=\sum_{j} w_{i j} \delta s_{j}$ with respect to time, use Eq (S7), and use the symmetric connectivity, to arrive at:

$$
\begin{aligned}
\delta \dot{J}_{i} & =\sum_{j} w_{i j} \delta \dot{s}_{j}=\sum_{j} w_{i j}\left(-\frac{1}{\tau_{s}} \delta s_{j}+\phi_{0, j}^{\prime} \delta J_{j}\right) \\
& =\left(-\frac{1}{\tau_{s}}+\sum_{j} w_{j i} \phi_{0, j}^{\prime}\right) \delta J_{j} \\
& =\left(K_{s}^{T} \delta \mathbf{J}\right)_{i} .
\end{aligned}
$$

If $\dot{\delta} s_{i}=0$ for all $i$, which is the case if $\delta s_{i}=e_{r}$ restricted to the first $N$ entries, we know that $\dot{\delta} \dot{J}_{i}=\sum_{j} w_{i j} \dot{\delta} \dot{s}_{j}=0$. Thus, in this restricted case, the left eigenvector proportional to $t_{i}=\delta J_{i}$ since it fulfills Eq (S3) restricted to the first $N \times N$ block.

We now consider again the full system (with facilitation and depression), where we start with the same Ansatz for parametrization of the variables $t_{i}{ }^{2}$

$$
t_{i} \equiv \sum_{j} w_{i j} \delta s_{j}
$$

and continue to find variables $v_{i}$ and $z_{i}$ that satisfy the full equations Eq (S3).

First, we differentiate Eq (S10) with respect to time and use the linear response Eq $\sqrt{13})$ to obtain

$$
\begin{aligned}
\dot{t_{i}} & =\sum_{j} w_{i j} \dot{\delta s_{j}}=\sum_{j} w_{i j}\left(-\frac{\delta s_{j}}{\tau_{s}}+\phi_{j}^{\prime} u_{0, j} x_{0, j} \sum_{k} w_{j k} \delta s_{k}+\phi_{0, j} x_{0, j} \delta u_{j}+\phi_{0, j} u_{0, j} \delta x_{j}\right) \\
& =-\frac{t_{i}}{\tau_{s}}+\sum_{j} w_{i j} u_{0, j} x_{0, j} \phi_{j}^{\prime} t_{j}+\sum_{j} w_{i j} \phi_{0, j} x_{0, j} \delta u_{j}+\sum_{j} w_{i j} \phi_{0, j} u_{0, j} \delta x_{j} .
\end{aligned}
$$

We then equate Eqs. (S4) and (S11), which yields the following identity:

$$
\sum_{j} w_{i j} \phi_{0, j}\left(x_{0, j} \delta u_{j}+u_{0, j} \delta x_{j}\right)=\sum_{j} w_{j i} \phi_{j}^{\prime}\left(U\left(1-u_{0, j}\right) v_{j}-u_{0, j} x_{0, j} z_{j}\right) .
$$

\footnotetext{
${ }^{2}$ This was motivated by numerical evaluations of the left eigenvector $e_{l}$ of the full system, which showed that here also $t_{i}=\delta J_{i}$
} 
To relate the remaining variables $\delta u_{i}$ and $\delta x_{i}$ to the new variables $v_{i}$ and $z_{i}$, we make a linear Ansatz:

$$
\begin{aligned}
& v_{i}=\alpha_{1} \delta u_{i}+\alpha_{2} \delta x_{i} \\
& z_{i}=\beta_{1} \delta u_{i}+\beta_{2} \delta x_{i}
\end{aligned}
$$

By differentiating these equations with respect to time and equating the result to Eqs. (S5) and (S6), respectively, we find the following equations

$$
\begin{aligned}
-\frac{v_{i}}{\tau_{u}}+\phi_{0, i} x_{0, i}\left(t_{i}-z_{i}\right)-U \phi_{0, i} v_{i} & =\alpha_{1} \delta \dot{u}_{i}+\alpha_{2} \delta \dot{x}_{i} \\
-\frac{z_{i}}{\tau_{x}}+u_{0, i} \phi_{0, i}\left(t_{i}-z_{i}\right) & =\beta_{1} \delta \dot{u}_{i}+\beta_{2} \delta \dot{x}_{i}
\end{aligned}
$$

The linear response for $\dot{u}_{i}$ and $\dot{x}_{i}$ can be obtained from Eq (13) (substituting $t_{i}=\sum_{j} w_{i j} \delta s_{j}$ ):

$$
\begin{aligned}
& \delta \dot{u}_{i}=-\delta u_{i}\left(\frac{1}{\tau_{u}}-U \phi_{0, i}\right)+U\left(1-u_{0, i}\right) \phi_{i}^{\prime} t_{i} \\
& \delta \dot{x}_{i}=-\delta x_{i}\left(\frac{1}{\tau_{x}}-\phi_{0, i}\right)-x_{0, i} \phi_{0, i} \delta u_{i}-u_{0} x_{0} \phi_{i}^{\prime} t_{i} .
\end{aligned}
$$

By inserting these two equations and Eqs. (S13) and $(\mathrm{S} 14)$ into Eqs. $(\mathrm{S} 15)$ and $(\mathrm{S} 16)$, we obtain two closed equations in $\delta u_{i}, \delta x_{i}, t_{i}$. By equating coefficients for $\delta u_{i}, \delta x_{i}, t^{3}$ we obtain solutions for the coefficients $\alpha_{1}, \alpha_{2}, \beta_{1}, \beta_{2}$ that fulfill these equations:

$$
\begin{aligned}
\alpha_{1} & =\frac{x_{0, i} \phi_{0, i}\left[U \tau_{u} \tau_{x} \phi_{0, i}+u_{0, i}\left(\tau_{x}-\tau_{u}\right)\right]}{U\left(1-u_{0, i}\right) \phi_{i}^{\prime}\left[\tau_{u} \tau_{x} \phi_{0, i}\left(U-u_{0, i}^{2}\right)+u_{0, i}\left(\tau_{x}-\tau_{u}\right)\right]} \\
\alpha_{2} & =-\frac{\tau_{u} \tau_{x} u_{0, i} \phi_{0, i}^{2}}{\phi_{i}^{\prime}\left[\tau_{u} \tau_{x} \phi_{0, i}\left(u_{0, i}^{2}-U\right)+u_{0, i}\left(\tau_{u}-\tau_{x}\right)\right]} \\
\beta_{1} & =-\frac{\tau_{u} \tau_{x} u_{0, i} \phi_{0, i}^{2}}{\phi_{i}^{\prime}\left(\tau_{u} \tau_{x} \phi_{0, i}\left(u_{0, i}^{2}-U\right)+u_{0, i}\left(\tau_{u}-\tau_{x}\right)\right)} \\
\beta_{2} & =\frac{u_{0, i} \phi_{0, i}\left[\tau_{u}\left(\tau_{x} \phi_{0, i}\left(U-u_{0, i}\right)-1\right)+\tau_{x}\right]}{x_{0, i} \phi_{i}^{\prime}\left[\tau_{u} \tau_{x} \phi_{0, i}\left(u_{0, i}^{2}-U\right)+u_{0, i}\left(\tau_{u}-\tau_{x}\right)\right]} .
\end{aligned}
$$

A little bit of further algebra shows that these coefficients together with Eqs. (S13) and (S14) also satisfy Eq (S12), as for every $j$ it holds that $\phi_{j}^{\prime}\left(U\left(1-u_{0, j}\right) v_{j}-u_{0, j} x_{0, j} z_{j}\right)=\phi_{0, j}\left(x_{0, j} \delta u_{j}+u_{0, j} \delta x_{j}\right)$.

Thus, we have found a linear parametrization

$$
\mathbf{y}^{\prime}(\mathbf{y})^{T}=\left((W \delta \mathbf{s})^{T},\left(\alpha_{1} \delta \mathbf{u}+\beta_{1} \delta \mathbf{x}\right)^{T},\left(\alpha_{2} \delta \mathbf{u}+\beta_{2} \delta \mathbf{x}\right)^{T}\right)
$$

which fulfills $\dot{\mathbf{y}}^{\prime}=K^{T} \mathbf{y}^{\prime}$. In addition, we know that if $\mathbf{y}=e_{r}$, then the original system dynamics vanish since $\dot{\mathbf{y}}=K \mathbf{y}=0$. Thus, since the parametrization is linear in the original variables, it also holds that $\dot{\mathbf{y}}^{\prime}=0$, and the parametrization satisfies Eq $\mathrm{S} 3$. This makes $\mathbf{y}^{\prime}\left(e_{r}\right)^{T}$ proportional to the (unique) left eigenvector $e_{l}$ of the 0-eigenvalue.

Finally, we can evaluate the vector $\mathbf{y}^{\prime}\left(e_{r}\right)$ by using Eq 14 in Eq (S18):

$$
\mathbf{y}^{\prime}\left(e_{r}\right)^{T}=\left({\frac{d \mathbf{J}_{0}}{d \varphi}}^{T},\left(\alpha_{1} \frac{d \mathbf{u}_{0}}{d \varphi}+\alpha_{2} \frac{d \mathbf{x}_{0}}{d \varphi}\right)^{T},\left(\beta_{1} \frac{d \mathbf{u}_{0}}{d \varphi}+\beta_{2} \frac{d \mathbf{x}_{0}}{d \varphi}\right)^{T}\right)
$$

Note, that in the first component we used that $\frac{d J_{0, i}}{d \varphi}=\sum_{j} w_{i j} \frac{d s_{0, j}}{d \varphi}$.

\footnotetext{
${ }^{3}$ Comparing coefficients of any two of the three variables yields 4 equations, which give the same solution and satisfy the equations posed by the remaining variable.
} 


\section{Normalization constant S}

In the last section we have found a vector $\mathbf{y}^{\prime T}$ proportional to the left eigenvector $e_{l}$. Since it holds that $e_{l} e_{r}=1$, it remains to calculate the normalization constant $S$ such this normalization condition is fulfilled by

$$
e_{l}=\frac{1}{S} \mathbf{y}^{\prime}\left(e_{r}\right)^{T} .
$$

First, we calculate the components of the vector $e_{r}$, Eq (14), using the steady-state values of Eq (12):

$$
\begin{aligned}
\frac{d s_{0, i}}{d \varphi} & =\tau_{s}\left(u_{0, i} x_{0, i} \phi_{0, i}^{\prime} \frac{d J_{0, i}}{d \varphi}+x_{0, i} \phi_{0, i} \frac{d u_{0, i}}{d \varphi}+u_{0, i} \phi_{0, i} \frac{d x_{0, i}}{d \varphi}\right), \\
\frac{d u_{0, i}}{d \varphi} & =u_{0, i}^{\prime} \phi_{0, i}^{\prime} \frac{d J_{0, i}}{d \varphi}=\frac{(1-U) U \tau_{u}}{\left(U \phi_{0, i} \tau_{u}+1\right)^{2}} \phi_{0, i}^{\prime} \frac{d J_{0, i}}{d \varphi}, \\
\frac{d x_{0, i}}{d \varphi} & =x_{0, i}^{\prime} \phi_{0, i}^{\prime} \frac{d J_{0, i}}{d \varphi}=-\frac{U \tau_{x}\left(\phi_{0, i} \tau_{u}\left(U \phi_{0, i} \tau_{u}+2\right)+1\right)}{\left(U \phi_{0, i}\left(\tau_{u}+\phi_{0, i} \tau_{u} \tau_{x}+\tau_{x}\right)+1\right)^{2}} \phi_{0, i}^{\prime} \frac{d J_{0, i}}{d \varphi},
\end{aligned}
$$

where we introduced the shorthand notations $u_{0, i}^{\prime}=\frac{d u_{0, i}}{d \phi_{0, i}}, x_{0, i}^{\prime}=\frac{d x_{0, i}}{d \phi_{0, i}}$ and $\phi_{0, i}^{\prime}=\left.\frac{d \phi_{i}}{d J_{i}}\right|_{J_{0, i}}$. We have also used the steady-state values of Eq (12) to calculate the values of $u_{0, i}^{\prime}$ and $x_{0, i}^{\prime}$ by differentiating with respect to $\phi_{0, i}$.

Now, using Eq. $(\mathrm{S} 20)$ in $e_{l} \cdot e_{r}=1$, and plugging in Eqs. (14) and (S19), we find that:

$$
\begin{aligned}
S= & \mathbf{y}^{\prime}\left(e_{r}\right)^{T} \cdot e_{r} \\
= & \tau_{s} \sum_{i} \frac{d J_{0, i}}{d \varphi}\left(u_{0, i} x_{0, i} \phi_{0, i}^{\prime} \frac{d J_{0, i}}{d \varphi}+x_{0, i} \phi_{0, i} \frac{d u_{0, i}}{d \varphi}+u_{0, i} \phi_{0, i} \frac{d x_{0, i}}{d \varphi}\right) \\
& +\sum_{i} \frac{d u_{0, i}}{d \varphi}\left(\alpha_{1} \frac{d u_{0, i}}{d \varphi}+\alpha_{2} \frac{d x_{0, i}}{d \varphi}\right)+\sum_{i} \frac{d x_{0, i}}{d \varphi}\left(\beta_{1} \frac{d u_{0, i}}{d \varphi}+\beta_{2} \frac{d x_{0, i}}{d \varphi}\right) \\
= & \tau_{s} \sum_{i}\left(\frac{d J_{0, i}}{d \varphi}\right)^{2} \phi_{0, i}^{\prime}\left(u_{0, i} x_{0, i}+x_{0, i} \phi_{0, i} u_{0, i}^{\prime}+u_{0, i} \phi_{0, i} x_{0, i}^{\prime}\right) \\
& +\sum_{i}\left(\frac{d J_{0, i}}{d \varphi}\right)^{2} \phi_{0, i}^{\prime 2}\left[\alpha_{1} u_{0, i}^{\prime 2}+\beta_{2} x_{0, i}^{\prime 2}+2 \alpha_{2} u_{0, i}^{\prime} x_{0, i}^{\prime}\right] \\
= & U \sum_{i} \frac{\left(\frac{d J_{0, i}}{d \varphi}\right)^{2} \phi_{i}^{\prime}}{\left[U \phi_{0, i}\left(\tau_{u}\left(\tau_{x} \phi_{0, i}+1\right)+\tau_{x}\right)+1\right]^{3}} \\
& {\left[\tau_{s}\left[\tau_{u} \phi_{0, i}\left(U \tau_{u} \phi_{0, i}+2\right)+1\right]\left[U \phi_{0, i}\left(\tau_{u}\left(\tau_{x} \phi_{0, i}+1\right)+\tau_{x}\right)+1\right]\right.} \\
& -\phi_{0, i}\left[(U-1) \tau_{u}^{2}+U \tau_{x}^{2}\left(\tau_{u} \phi_{0, i}+1\right)\left(\tau_{u} \phi_{0, i}\left(U \tau_{u} \phi_{0, i}+2\right)+1\right)\right] \\
& \left.-\frac{(U-1) U \tau_{u}^{2} \tau_{x} \phi_{0, i}\left(\tau_{u} \phi_{0, i}+1\right)}{\left(U \tau_{u} \phi_{0, i}+1\right)}\right],
\end{aligned}
$$

which defines the normalization constant $S$. In the last equation we used the steady-state values of Eq (12) to calculate the values of $u_{0, i}^{\prime}$ and $x_{0, i}^{\prime}$ by differentiating with respect to $\phi_{0, i}$. Additionally, the coefficients of Eq (S17) were used. 


\section{Diffusion strength B}

To calculate the correlation function of Eq $\sqrt{22})$, we first note that only terms $\left\langle\eta_{i}(t) \eta_{i}(t+\tau)\right\rangle=\delta(\tau)$ remain in expectation. Thus, we arrive at:

$$
\begin{aligned}
\langle\dot{\varphi}(t) \dot{\varphi}(t+\tau)\rangle= & \left\langle e_{l} L(t) e_{l} L(t+\tau)\right\rangle \\
= & \sum_{i=1}^{n}\left(e_{l, i}^{2} u_{0, i}^{2} x_{0, i}^{2}+e_{l, n+i}^{2} U^{2}\left(1-u_{0, i}\right)^{2}+e_{l, 2 n+i}^{2} u_{0, i}^{2} x_{0, i}^{2}\right) \phi_{0, i} \delta(\tau) \\
& +2 \sum_{i=1}^{n}\left[\left(e_{l, i} e_{l, n+i}-e_{l, n+i} e_{l, 2 n+i}\right) U\left(1-u_{0, i}\right)-e_{l, i} e_{l, 2 n+i} u_{0, i} x_{0, i}\right] u_{0, i} x_{0, i} \phi_{0, i} \delta(\tau) \\
= & \frac{1}{S^{2}} \sum_{i}\left(\frac{d J_{0, i}}{d \varphi}\right)^{2} \phi_{0, i}\left[u_{0, i}^{2} x_{0, i}^{2}+U^{2}\left(1-u_{0, i}\right)^{2} \phi_{0, i}^{\prime 2}\left(\alpha_{1} u_{0, i}^{\prime}+\alpha_{2} x_{0, i}^{\prime}\right)^{2}\right. \\
& \left.+u_{0, i}^{2} x_{0, i}^{2} \phi_{0, i}^{\prime 2}\left(\beta_{1} u_{0, i}^{\prime}+\beta_{2} x_{0, i}^{\prime}\right)^{2}\right] \delta(\tau) \\
& +\frac{2}{S^{2}} \sum_{i}\left(\frac{d J_{0, i}}{d \varphi}\right)^{2} \phi_{0, i} u_{0, i} x_{0, i} U\left(1-u_{0, i}\right) \\
& -\frac{2}{S^{2}} \sum_{i}\left(\frac{d J_{0, i}}{d \varphi}\right)^{2} \phi_{0, i} u_{0, i}^{2} x_{0, i}^{2} \phi_{0, i}^{\prime}\left(\beta_{1} u_{0, i}^{\prime}+\beta_{2} x_{0, i}^{\prime}\right) \delta(\tau) \\
= & \frac{U^{2}}{S^{2}} \sum_{i} \frac{\left(\frac{d J_{0, i}}{d \varphi}\right)^{2} \phi_{0, i}\left(1+2 \tau_{u} \phi_{0, i}+U \tau_{u}^{2} \phi_{0, i}^{2}\right)^{2}}{\left(U \phi_{0, i}\left(\tau_{u}\left(\tau_{x} \phi_{0, i}+1\right)+\tau_{x}\right)+1\right)^{4}} \delta(\tau) \\
\equiv & B \delta(\tau) .
\end{aligned}
$$

In the last equation we again have used the steady-state values of Eq 12 to calculate the values of $u_{0, i}^{\prime}$ and $x_{0, i}^{\prime}$ by differentiating with respect to $\phi_{0, i}$. Additionally, the coefficients of Eq S17) were used.

\section{Drift term}

Left-multiplying Eq. 25] by the left eigenvector $e_{l}$ yields:

$$
\begin{aligned}
\dot{\varphi}=e_{l}\left(\begin{array}{c}
\mathbf{x}_{0} \mathbf{u}_{0} \Delta \vec{\phi}(\varphi) \\
U\left(1-\mathbf{u}_{0}\right) \Delta \vec{\phi}(\varphi) \\
-\mathbf{x}_{0} \mathbf{u}_{0} \Delta \vec{\phi}(\varphi)
\end{array}\right)+\sqrt{B} \eta \\
=\frac{1}{S} \sum_{i}\left[\frac{d J_{0, i}}{d \varphi} x_{0, i} u_{0, i}+\left(\alpha_{1} \frac{d u_{i}}{d \varphi}+\alpha_{2} \frac{d x_{i}}{d \varphi}\right) U\left(1-u_{0, i}\right)\right. \\
\left.-\left(\beta_{1} \frac{d u_{i}}{d \varphi}+\beta_{2} \frac{d x_{i}}{d \varphi}\right) x_{0, i} u_{0, i}\right] \Delta \phi_{i}(\varphi)+\sqrt{B} \eta \\
=\frac{U}{S} \sum_{i} \frac{d J_{0, i}}{d \varphi} \frac{1+\tau_{u} \phi_{0, i}\left(U \tau_{u} \phi_{0, i}+2\right)}{\left(U \phi_{0, i}\left(\tau_{u} \tau_{x} \phi_{0, i}+\tau_{u}+\tau_{x}\right)+1\right)^{2}} \Delta \phi_{i}(\varphi)+\sqrt{B} \eta
\end{aligned}
$$

where we have used Eqs. (S22) and (S23), as well as Eq (S17) in the last equality. 


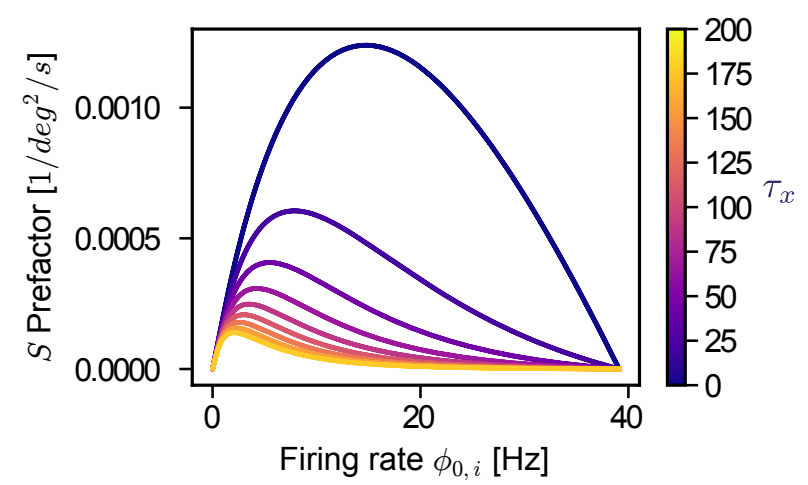

Fig S1-1. Pre-factor of normalizer for vanishing facilitation. Pre-factor $\frac{d J_{0, i}{ }^{2}}{d \varphi} \phi_{0, i}^{\prime} \frac{1}{\left(1+\phi_{0, i} \tau_{x}\right)^{3}}$ for varying firing rates $\phi_{0, i}$ and depression time constant $\tau_{x}$. Color legend on the right hand side shows values of $\tau_{x}$ in units of ms.

\section{Critical depression time constant}

To analyze the vanishing normalization constant for growing depression time constant $\tau_{x}$ (see Supplementary Figure S8 Fig), we set $U=1$ in the normalization constant $S$ (Eq (19) of the main text), which yields:

$$
S=\sum_{i} \frac{d{J_{0, i}}^{2}}{d \varphi} \phi_{0, i}^{\prime} \frac{\left(\phi_{0, i} \tau_{s} \tau_{x}-\phi_{0, i} \tau_{x}^{2}+\tau_{s}\right)}{\left(1+\phi_{0, i} \tau_{x}\right)^{3}} .
$$

Inspecting Eq (S28), we find that $S$ will be crossing to zero if $\phi_{0, i} \tau_{s} \tau_{x}-\phi_{0, i} \tau_{x}^{2}+\tau_{s} \leq 0$ for many summands. Solving this at equality for $\tau_{x}$ yields a single positive solution

$$
\tau_{x, i}=\frac{1}{2}\left(\tau_{s}+\sqrt{\frac{\tau_{s}\left(\phi_{0, i} \tau_{s}+4\right)}{\phi_{0, i}}}\right),
$$

which approaches $\tau_{s}$ for large firing rates $\phi_{0, i} \rightarrow \infty$. Thus, summands of Eq S28 with larger firing rates $\phi_{0, i}$ will be the first to turn negative if $\tau_{x}>\tau_{s}$. As $\tau_{x}$ grows further, also summands with smaller firing rates will become negative according to Eq $\sqrt{\mathrm{S} 29}$, , eventually turning the total sum to zero. On the other hand, the range of firing rates that contribute at all to $\mathrm{Eq}(\mathrm{S} 28)$ is limited to the flanks of the firing rate profile: the pre-factors $\frac{d J_{0, i}{ }^{2}}{d \varphi} \phi_{0, i}^{\prime}$ in Eq (S28) will vanish for $\phi_{0, i} \rightarrow 0$ and $\phi_{0, i} \rightarrow \max _{i} \phi_{0, i}$, since in both cases $\frac{d J_{0, i}}{d \varphi}=0$. This interplay of single summands turning negative and their contributing only in bump-shape dependent ranges is hard to generally analyze further. However, the relation found in Eq (S29) is valid for any bump system with depression, and will eventually lead to a vanishing normalization factor $S$.

We will now resort to a numerical analysis for the spiking system used in the main text. Plotting the pre-factor $\frac{d J_{0, i}{ }^{2}}{d \varphi} \phi_{0, i}^{\prime} \frac{1}{\left(1+\phi_{0, i} \tau_{x}\right)^{3}}$ against the firing rate $\phi_{0, i}$ for varying $\tau_{x}$ (see Fig S1-1 ), we see that as $\tau_{x}$ increases beyond $50 \mathrm{~ms}$, the range of firing rates with positive contributions to the sum quickly decays to between 0 and $15 \mathrm{~Hz}$, with maxima between $2 \mathrm{~Hz}$ and $5.5 \mathrm{~Hz}$. Evaluating Eq $(\mathrm{S} 29)$ for $\tau_{s}=100 \mathrm{~ms}$ at these firing rates, the corresponding values of $\tau_{x, i}$ in Eq $\mathrm{S} 29$ ) are $279.1 \mathrm{~ms}$ (for $2 \mathrm{~Hz}$ ) and $193.8 \mathrm{~ms}$ (for $5.5 \mathrm{~Hz}$ ). This only yields an estimate of the range in which summands will switch to negative values, and not the value of the total sum in Eq (S28). A numerical solution of Eq $(\mathrm{S} 28)$ yields the depression time constant at which Eq S28 becomes zero: $\tau_{x}, c=223.9 \mathrm{~ms}$, which nevertheless lies in the thus estimated range. 


\section{References}

1. Burak Y, Fiete IR. Fundamental Limits on Persistent Activity in Networks of Noisy Neurons. Proceedings of the National Academy of Sciences. 2012;109(43):17645-17650. 